\title{
Grating Lobe Suppression in Rotationally Tiled Arrays
}

\author{
Abdullah Alshammary ${ }^{1,2}$, Stephan Weiss ${ }^{1}$, and Sultan Almorqi ${ }^{2}$ \\ ${ }^{1}$ Department of Electronic \& Electrical Engineering, University of Strathclyde, Glasgow, Scotland \\ ${ }^{2}$ KACST, Riyadh, Saudi Arabia \\ \{Abdullah.Alshammary, Stephan.Weiss\}@ strath.ac.uk; salmorqi@kacst.edu.sa
}

\begin{abstract}
Uniform placement of array elements limits its maximum frequency due to the formation of grating lobes. While non-uniform element or subarray spacing have significantly lower grating lobes, it reduces aperture efficiency and leads to arrays that are difficult to design and manufacture. We propose a modular asymmetric convex-shaped subarray to construct the array by rotation and translation, filling the aperture without overlaps or gaps. This new approach can achieve lower grating lobes compared to uniform array geometries. It can also lower design, manufacturing and operation costs by offering interoperable subarrays and provide array size flexibility.

Index Terms - subarray, grating lobes, plane tiling, tessella-
\end{abstract} tion

\section{INTRODUCTION}

When constructing sensor arrays for beamforming, a maximum spacing between sensor elements of less than half the wavelength of the observed signal is usually required to avoid spatial aliasing. If the minimum elements spacings of uniform arrays is wider, spatial aliasing occurs, manifested by the grating lobes that appear in the array response. For wideband arrays however, increasing the upper frequency limit will require smaller elements spacing leading to increased risk of mutual coupling. Also, in this case a higher number sensor elements is required to satisfy a fixed aperture size.

Many solutions have been suggested towards grating lobe reduction. Thinned array [1], where random array elements are removed, can maintain the original beamwidth but the gain decreases and sidelobe level increases because of the reduced total number of elements per unit area combined with inefficient aperture illumination.

In this paper, we consider the constructing larger array apertures from smaller subarrays is a popular design technique particularly in the context of radar [2]. This is for example utilised in architectures of narrowband subarrays followed by a time delay. This is referred to as a subarray structure, and has been addressed e.g. in [4], [5], [6], [2], [3]. The general problem that has been researched is the tiling of the subarrays in order to minimize quantization sidelobes [4], [5], [6]. Sometimes also the narrowband beamforming weights are optimized in order to suppress sidelobes in the beamformer's broadband response [4], [7].

Grating lobe suppression has also been applied to subarrays. At subarray level, the grating lobes are reduced but not eliminated since each subarray output still suffers from grating lobes that equal the level of the main beam. However, the subarray position or shape ensures that grating lobes do not necessarily coincide. One common subarray approach is to construct subarrays with random shapes [8]. Another solution is to slightly twist the subarray by different angles [9], [10] or displace their location in one dimension [11] or two dimensions [9]. These techniques likely create a challenge in the array design and manufacturing, because random subarray outlines and sizes require unique components and programming for each subarray. The distribution network and subarray processing will also need tailoring to suit each individual subarray.

Therefore, in this paper we propose to utilise a single subarray shape, which can be used to construct the entire array by translation and rotation. This isohedral design can be based on known pentagonal or hexagonal tiles that can densely fill a plane without gaps or overlapping. To motivate and demonstrate this design, in the following we will first analyse grating lobes in Sec. II. Introduce plane tiling and the limitations to array and subarray design in Sec. III. In Sec. IV an example of a pentagonal subarray and aperiodic design is compared to a uniform circular array with an equal number of array elements. Finally, conclusions are drawn in Sec. V.

\section{ANALYSIS OF GRATING LOBES}

\section{A. Grating Lobes in Uniform Arrays}

Consider a rectangular array with uniform spacing $d$ lying on the $x-y$ plane. An incident signal with frequency $\omega$ from azimuth angle $\varphi$ and elevation angle $\vartheta$ is characterized by the wave number vector [12] as

$$
\mathbf{k}=\frac{\omega}{c}\left[\begin{array}{c}
\sin \vartheta \cos \varphi \\
\sin \vartheta \sin \varphi
\end{array}\right]
$$

and the array gain in response to the wavenumber vector $\mathrm{k}$ is

$$
P(\omega, \vartheta, \varphi)=\sum_{n_{x}=0}^{N_{x}-1} \sum_{n_{y}=0}^{N_{y}-1} w\left[n_{x}, n_{y}\right] e^{-j \mathbf{x}^{\mathrm{H}}\left(n_{x}, n_{y}\right) \mathbf{k}},
$$

where $\mathbf{x}^{\mathrm{H}}\left(n_{x}, n_{y}\right)$ are the coordinates and $w\left[n_{x}, n_{y}\right]$ the weights of a sensor element indexed by $n_{x}$ and $n_{y}, n_{x}, n_{y} \in$ $\mathbb{N}$.

For a uniformly spaced, square and symmetric array, with nearest element spacing $d$, we have

$$
\mathbf{x}\left(n_{x}, n_{y}\right)=d\left[\begin{array}{l}
n_{x}-\frac{N_{x}-1}{N_{y}^{2}} \\
n_{y}-\frac{N_{y}}{2}
\end{array}\right] .
$$


The array weights $w\left[n_{x}, n_{y}\right]$ can scale the array response or shift its phase, but have no effect on the grating lobe separation w.r.t. the main lobe. Assuming that the weights attached to each element perform phase shifts, the array is then steered towards the direction $\mathbf{k}_{0}\left(\omega_{0}, \vartheta_{0}, \varphi_{0}\right)$. With this, and the array configuration in (3), the array gain in (2) of the array steered towards the direction $\mathbf{k}_{0}\left(\omega_{0}, \vartheta_{0}, \varphi_{0}\right)$ simplifies to

$$
P(\omega, \vartheta, \varphi)=\frac{1}{\sqrt{N_{x} N_{y}}} \frac{\sin \left(\frac{1}{2} \mathbf{x}_{\max }^{\mathrm{H}} \Delta \mathbf{k}\right)}{\sin \left(\frac{1}{2} \mathbf{x}_{\min }^{\mathrm{H}} \Delta \mathbf{k}\right)},
$$

where $\Delta \mathbf{k}=\mathbf{k}-\mathbf{k}_{0}$ and $\mathbf{x}_{\min }=\left[\begin{array}{ll}d & d\end{array}\right]^{\mathrm{H}}$ and $\mathbf{x}_{\max }=$ $d\left[\begin{array}{ll}N_{x} & N_{y}\end{array}\right]^{\mathrm{H}}$. The array response is periodic w.r.t. wave number vector $\Delta \mathbf{k}$ with period $\frac{2 \pi}{d}$. As a result, the grating lobes are located at zeros of the denominator in the equation above, i.e. $\Delta \mathbf{k}=p \frac{2 \pi}{d} \quad \forall p \in \mathbb{Z}$ in both $x$ - and $y$-axes.

\section{B. Grating Lobes in Rotationally Tiled Arrays}

Array with suppressed grating lobes can be constructed with isohedral subarrays. Isohedral tiling is a plane tiling based on a single shape of tile, where the overall array can be constructed by placing rotated and translated subarrays. While translation does not affect the subarray response w.r.t. its phase center, rotation changes the azimuth angle of arrival by an amount equal to the rotation angle $\psi$. For a rotationally tiled array with $M$ subarrays and an order of rotation, $L,-$ to be further elaborated in Sec. III — the $M$ subarrays will be rotated at $L$ different angles.

If the array is steered towards $\mathbf{k}_{0}\left(\omega_{0}, \vartheta_{0}, \varphi_{0}\right)$, the gain of a rotationally tiled array w.r.t. a signal characterised by the wave number $\mathbf{k}(\omega, \vartheta, \varphi)$ is

$$
P(\omega, \vartheta, \varphi)=\frac{1}{\sqrt{N_{x} N_{y}}} \sum_{l=0}^{L-1} \sum_{m \in M_{l}} \frac{\sin \left(\frac{1}{2} \mathbf{x}_{\max }^{\mathrm{H}} \Delta \mathbf{k} \cos \psi_{l}\right)}{\sin \left(\frac{1}{2} \mathbf{x}_{\min }^{\mathrm{H}} \Delta \mathbf{k} \cos \psi_{l}\right)},
$$

where again $\Delta \mathbf{k}=\mathbf{k}-\mathbf{k}_{0}$, and $M_{l}$ is the number of subarrays that share the same rotation angle $\psi_{l}$.

Each subarray group $m \in M_{l}$ has a periodic response with period $\Lambda=2 \pi\left(d \cos \psi_{l}\right)^{-1}$ and grating lobes forming at $\Delta \mathbf{k}_{l}=p \Lambda \quad \forall p= \pm 1, \pm 2, \cdots$ in both $x$ and $y$ axis directions. The sum of periodic responses is also periodic with a period equal to the least common multiple. Hence as the order of rotation $L$ increases, full grating lobes, where the grating lobes of all subarrays coincide, will have a lower frequency of occurence.

\section{Plane Tiling in Array Design}

\section{A. Tiling}

In geometry, tiling or tessellation is the problem of finding a countable family of shapes that can tile a plane without overlap or gaps [13]. The closed set of tiles that can fill the plane in such a fashion is defined as

$$
\mathfrak{T}=\left\{T_{1} \cdots, T_{m}, \cdots T_{M}\right\} .
$$

Specifically, isohedral tiling allows only congruent tiles $T_{1} \cdots, T_{m}, \cdots T_{M}$ to populate the array aperture. Tile shapes

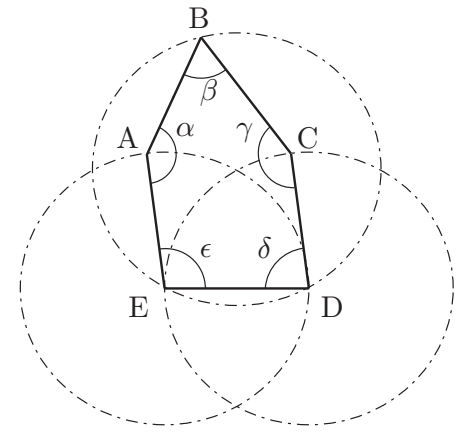

Fig. 1. Type 1 equilateral pentagon with $\delta+\epsilon=180^{\circ}$. Corners A, B and C can be moved along the circles while maintaining parallel sides $\mathrm{AE}$ and $\mathrm{CD}$. $\mathrm{ABC}$ is an equilateral triangle and $\mathrm{ACDE}$ is a rhombus.

are limited to convex polygons as will be explained in the next sub-section. The subarray outline is defined by the tile shape. Antenna elements are represented by the array lattice enclosed by the subarray outline and subject to the direct isometries, i.e. translation and rotation operations only, applied to the tile.

\section{B. Subarray Limitations}

All triangles and quadrilaterals can tile a plane [14]. But there are only 15 convex pentagon and three hexagons that can tile a plane without gap or overlap [15], and there are no known convex polygons beyond hexagons that can tile a plane.

When placing tiles of subarrays, the only permitted transformation to fit them in place are rotation and translation. Reflective operations are are known in the tiling literature but are not applicable to subarray tiles due to aperture orientation. Hence, only a limited number of patterns in the literature characterised by their notation $p n 11$ for $n=2,3, \ldots 6$ are applicable to subarrays indicating non-reflective configuration. The parameter $n$ is called the order of rotation (referred to as $L$ in Sec. II above) and it is the number of angles by which the base tile $T_{1}$ has to be rotated in a design.

Non-rotational design, where $n=1$ in $p 111$, have regular or equilateral polygons with uniform subarray spacing and will suffer from high grating lobes due to high rotational symmetry.

\section{EXAmple: EQuilateral PENTAgOn VS. UNifORM CIRCUlar ARRAY}

The tiling concept for subarrays is now demonstrated using an aperiodic pattern suggested by [14] based on a type 1 equilateral pentagon as described in [16]. Shown in Fig. 1, this equilateral pentagon has interior angles of $\alpha=140^{\circ}$, $\beta=60^{\circ}, \gamma=160^{\circ}, \delta=80^{\circ}$ and $\epsilon=100^{\circ}$ respectively.

In Fig. 2, 18 pentagons of the type shown in Fig. 1 construct an approximately circular array. Each subarray contains 42 array elements arranged on a square lattice, whereby the element spacing $d$ is half the wavelength at the operating frequency $f_{c}$. Therefore, across the 18 subarrays the total number of array elements of 756. The tiled array requires a small separation between subarrays as seen in Fig. 2 to account for subarray boundaries. Also the tiled array circumference is 


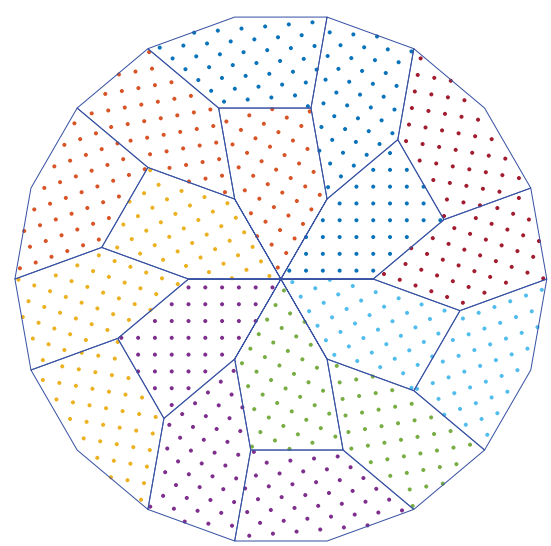

Fig. 2. Tiled array constructed by rotation and translation of 18 pentagon subarray tiles each containing 42 sensors elements each contained within an outer circle of radius $15.6 d$ where $d$ is the elements spacing.

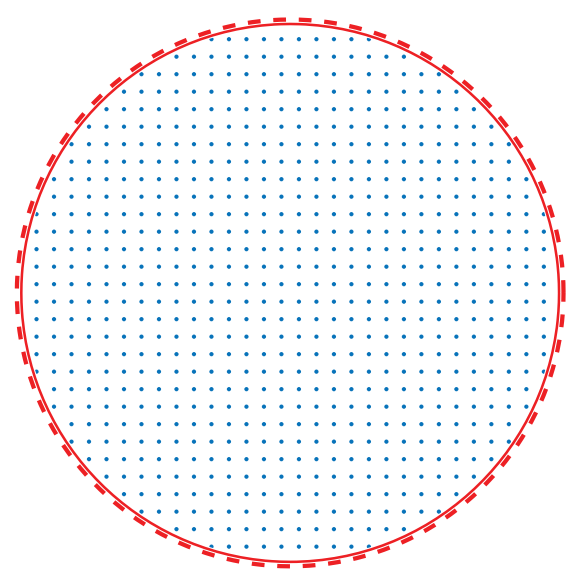

Fig. 3. Uniform circular array containing 756 elements - the same number of elements as the tiled array. Solid and dashed circles circumscribe the uniform circular array and the tiled array, respectively.

a series of straight edges, and does not constitute a perfect circle. It can, however, be inscribed into a circle of radius $15.6 d$.

We compare the tiled array above with a uniform circular array where sensor elements are placed on a square grid filling a circle as shown in Fig. 3, containing the same number of elements as the tiled configuration of Fig. 2. Because of the lack of internal boundaries, the circular array has a smaller diameter than the tiled array. In this example, the uniform circular array of Fig. 3 has a radius of $15.4 d$ compared to $15.6 d$ for the tiled array, if both arrays are circumscribed by circle.

Fig. 4 demonstrates the gain response of the uniform circular array when required to operate beyond the maximum frequency, such that the element spacing is now more than half the signal's wavelength. This can occur either due to the desired to space elements further apart in order to avoid mutual

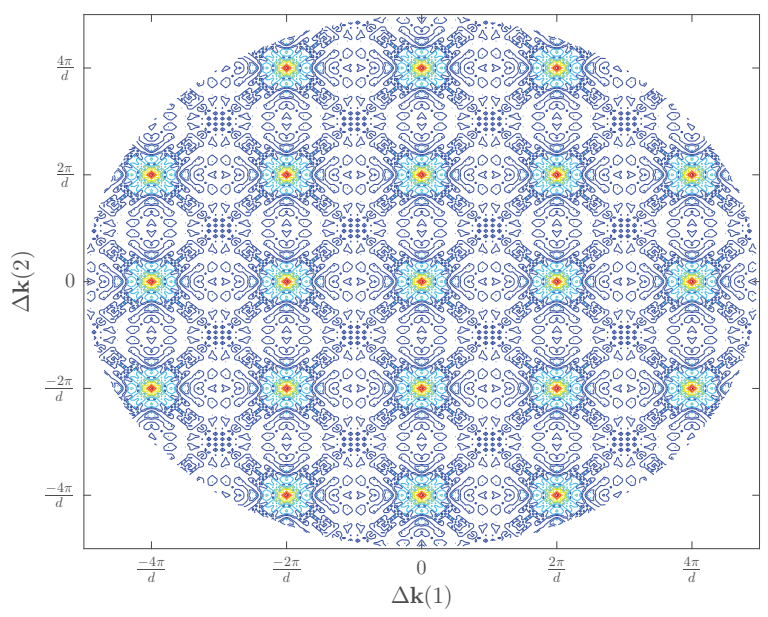

Fig. 4. Gain response of uniform circular array showing grating lobes.

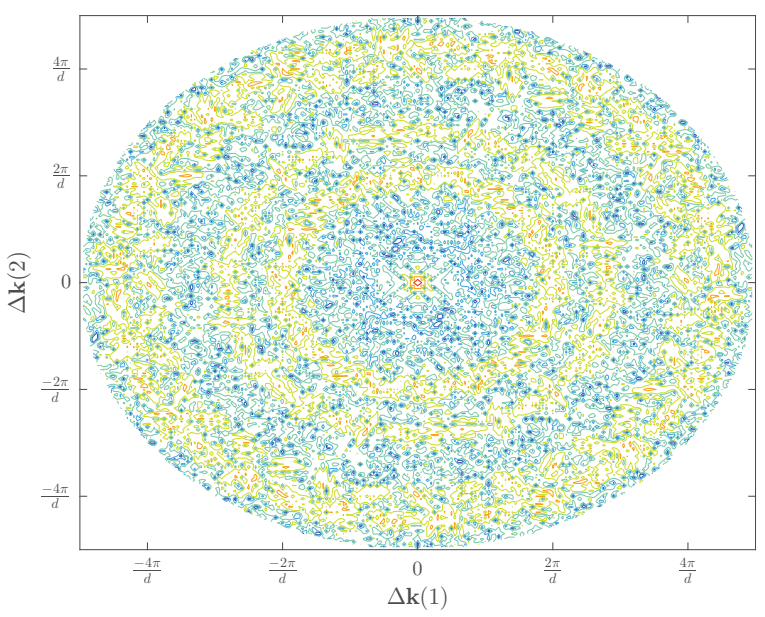

Fig. 5. Gain response of tiled array showing reduced grating lobes.

coupling, or to operate with a larger aperture but utilised a lower number of elements. With the main lobe centred in the diagram, for the uniform circular array in this case spatial aliasing occurs. As calculated for the uniformally spaced array with square element lattice in (4), this leads to the grating lobes seen in Fig. 4, which appear at integer multiples of $\frac{2 \pi}{d}$ in both $u$ and $v$ directions.

The gain response of the tiled array in Fig. 5 shows the sum of contribution of the individual subarrays, where grating lobes do not add constructively. The distance between minor grating lobes and the main lobe is unchanged at $\frac{2 \pi}{d}$ because the elements spacing is the same across subarrays, but the subarrays rotation by angles $\psi_{l}$ as in equation (5) causes grating lobes to rotate by the same angles around the mainlobe.

To compare the two gain responses directly, a cross-section for the first component of $\Delta \mathbf{k}$ for both the uniform cicular and proposed tiled array are shown in Fig. 6. The graphs show the grating lobes of the uniform circular array with a periodicity of $\frac{2 \pi}{d}$ and having the same $0 \mathrm{~dB}$ level as the main lobe at the origin. In contrast, the gain response of the proposed tiled 


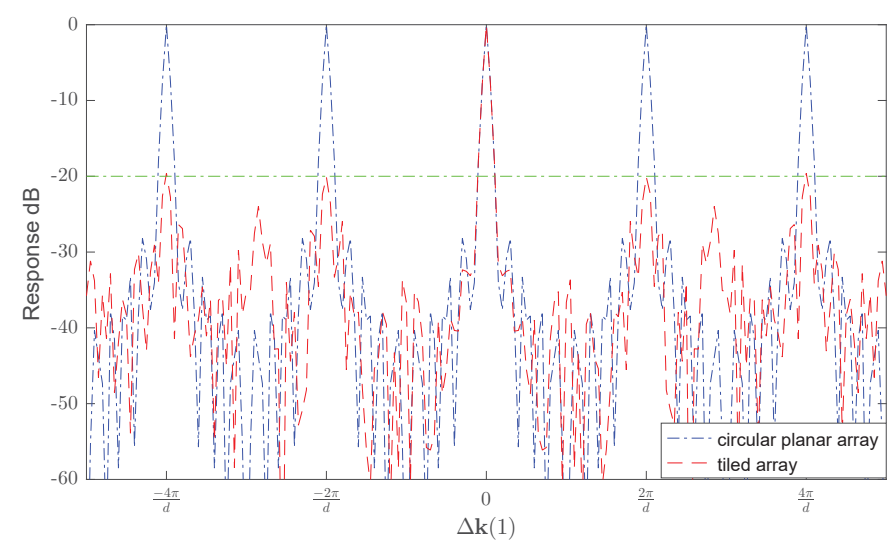

Fig. 6. Gain response along the $u$ domain of both the circular planar array and the tiled array.

TABLE I

COMPARISON SUMMARY BETWEEN THE TILED ARRAY AND THE UNIFORM CIRCULAR ARRAY WITH SAME NUMBER OF ELEMENTS.

\begin{tabular}{|l|c|c|}
\hline property & tiled array & circular planar array \\
\hline \hline number of elements & 756 & 756 \\
\hline diameter & $10.85 \lambda$ & $8.25 \lambda$ \\
\hline grating lobe level & $0 \mathrm{~dB}$ & $-20 \mathrm{~dB}$ \\
\hline beamwidth & $7^{\circ}$ & $7^{\circ}$ \\
\hline directivity & $30.6 \mathrm{dBi}$ & $30.7 \mathrm{dBi}$ \\
\hline
\end{tabular}

array demonstrates a reduced grating lobe level at $-20 \mathrm{~dB}$, while the width of the main lobe is approximately the same for the benchmark design. The summary of characteristics for both arrays is compiled in Tab. I.

As the analysis in Sec. II indicated, the grating lobes of the tiled array reduce as the number of design rotation angles increases. However, high rotational symmetry of the subarray or the element lattice may cause grating lobes from subarrays to coincide. In the Fig. 6, the tiled array response is suppressed by $-20 \mathrm{~dB}$ or to one tenth of the mainlobe level. This can be explained by the rotational symmetry of the square lattice w.r.t. integer multiples of $90^{\circ}$, which includes the design angle of $3 \beta=180^{\circ}$. This means that grating lobes of two differently rotated subarrays can coincide at the same angle. Hence, the cardinality of different orientations of the sensor element grid in Fig. 2 is 9, and therefore grating lobe suppression is expected to be at around $\frac{1}{9}$.

Table I summarizes the comparison between the uniform circular array in Fig. 3 and the proposed tiled array in Fig. 2. The grating lobes have reduced significantly while the directivity and beamwidth are unchanged. This expected because both directivity and beamwidth depends only on the number of sensors and their separation only.

\section{Conclusion}

Utilising rotational tiling in array design can introduce variance in both subarray phase center positions and sensors locations. This variance results from rotationally asymmetric subarrays being rotated and stacked in a pattern that leaves no gaps or overlaps. Rotationally tiled subarrays can significantly reduce grating lobes by breaking the alignment of individual subarray grating lobes. It however increases the array size due to the potentially irregular outer edges of the array and the clearance required for subarray boundaries, although a proposed pentagonal design leads to an approximatly circular configuration.

Compared to a large array with the same number of sensor elements arranged on a regular grid, the beamwidth and directivity of the tiled design are nearly unaffected, because aperture and number of elements are comparable. The tiling approach is shown to suppress grating lobes for spatially under-sampled arrays because grating lobes of subarrays do not necessarily add up constructively. Therefore, it is possible to design arrays with element spacing wider than half the wavelength - be it to reduce mutual coupling between sensor elements, or to operate an existing array at wider bandwidth without incurring spatial ambiguity.

\section{REFERENCES}

[1] R. Mailloux, Phased Array Antenna Handbook, ser. Antennas and Propagation Library. Artech House, 2005.

[2] W.-D. Wirth, Radar Techniques Using Array Antennas, ser. Radar, Sonar, Navigation and Avionics. Institution of Engineering and Technology, 2013.

[3] A. Alshammary and S. Weiss, "Low-Cost and Accurate Broadband Beamforming Based on Narrowband Sub-Arrays", in International ITG Workshop on Smart Antennas, Munich, Germany, March 2016.

[4] R. L. Haupt, "Optimized weighting of uniform subarrays of unequal sizes," IEEE Transactions on Antennas and Propagation, vol. 55, no. 4, pp. 1207-1210, April 2007.

[5] R. J. Mailloux, "Subarray technology for time delayed scanning arrays," in IEEE International Conference on Microwaves, Communications, Antennas and Electronics Systems, pp. 1-6, 2009.

[6] Z.-Y. Xiong, Z.-H. Xu, S.-W. Chen, and S.-P. Xiao, "Subarray partition in array antenna based on the algorithm x," IEEE Antennas and Wireless Propagation Letters, vol. 12, pp. 906-909, 2013.

[7] S. Somasundaram, "Wideband robust capon beamforming for passive sonar," IEEE Journal of Oceanic Engineering, vol. 38, no. 2, pp. 308322, April 2013.

[8] C. Tarran, M. Mitchell, and R. Howard, "Wideband phased array radar with digital adaptive beamforming," in IEE Colloquium High Resolution Radar and Sonar (Ref. No. 1999/051), pp. 1/1-1/7, 1999.

[9] Y. Krivosheev and A. Shishlov, "Grating lobe suppression in phased arrays composed of identical or similar subarrays," in IEEE International Symposium on Phased Array Systems and Technology, pp. 724-730, October 2010.

[10] V. D. Agrawal, "Grating-lobe suppression in phased arrays by subarray rotation," Proceedings of the IEEE, vol. 66, no. 3, pp. 347-349, March 1978.

[11] H. Wang, D. G. Fang, and Y. L. Chow, "Grating lobe reduction in a phased array of limited scanning," IEEE Transactions on Antennas and Propagation, vol. 56, no. 6, pp. 1581-1586, June 2008.

[12] H. L. Van Trees, Optimum Array Processing (Detection, Estimation, and Modulation Theory, Part IV), 1st ed. Wiley-Interscience, March 2002.

[13] B. Grünbaum and G. C. Shephard, Tilings and Patterns. New York, NY, USA: W. H. Freeman \& Co., 1986.

[14] M. Hirschhorn and D. Hunt, "Equilateral convex pentagons which tile the plane," Journal of Combinatorial Theory, Series A, vol. 39, no. 1, pp. $1-18,1985$.

[15] E. W. Weisstein, "Pentagon tiling," A Wolfram Web Resource., 2015. [Online]. Available: mathworld.wolfram.com/PentagonTiling

[16] D. Schattschneider, "Tiling the plane with congruent pentagons," Math ematics Magazine, vol. 51, pp. 29-44, 1978. 